\title{
Overview of Experimental Results on HL-2A
}

X.R.Duan, X.T.Ding, J.Q.Dong, Q.W.Yang, L.W.Yan, Yi Liu, X.L.Zou+, D.Q.Liu, W.M.Xuan, L.Y.Chen, J.Rao, X.M.Song, W.C.Mao, Q.M.Wang, Z.Cao, B.Li, J.Y.Cao, G.J.Lei, J.H.Zhang, X.D.Li, S.J.Wang, A.D.Liu*, M.N.Bu, Y.H.Chen, W.Chen, J.Cheng, C.H.Cui, Z.Y.Cui, Z.C.Deng, Y.B.Dong, B.B.Feng, Q.D.Gao, X.Y.Han, W.Y.Hong, H.T.Hu, M.Huang, Y.Huang, X.Q.Ji, Z.H.Kang, T.Lan*, G.S.Li, H.J.Li, Qing Li, Qiang Li, W.Li, Y.G.Li, Z.J.Li, Z.T.Liu, C.W.Luo, X.H.Mao, Y.D.Pan, J.F.Peng, K.Shao, X.Y.Song, H.J.Sun, A.K.Wang, H.Wang, M.X.Wang, Y.Q.Wang, Z.T.Wang. W.W.Xiao, Z.G.Xiao, Y.F.Xie, L.H.Yao, L.Y.Yao, D.L.Yu, B.S.Yuan, K.J.Zhao, Y.Z.Zheng, G.W.Zhong, C.P.Zhou, J.Zhou, Y.Zhou, J.C.Yan, C.X.Yu*, C.H.Pan and Yong Liu

\author{
Southwestern Institute of Physics, Chengdu, China \\ * University of Science and Technology of China, Hefei, China \\ + CEA, IRFM, Cadarache, France \\ E-mail of main author: duanxr@swip.ac.cn
}

\begin{abstract}
Significant experimental advances have been made on HL-2A tokamak along with substantial improvement and development of hardware. The three dimensional spectral structures of the low frequency zonal flow, the geodesic acoustic mode (GAM), and quasi-mode-like low frequency fluctuations have been observed simultaneously for the first time. In addition, the spectral structure of the density fluctuation at GAM frequency is also identified. A spontaneous particle transport barrier has been observed in Ohmic discharges without any external momentum input. The barrier is evidenced by particle perturbation study using modulated SMBI and microwave reflectometry. The non-local transport effect with new features induced by SMBI has been investigated. The e-fishbone instability excited by energetic electrons of non-Maxwellian distribution has been investigated via 10-channel CdTe hard x-ray detector. It is found that the e-fishbone is correlated with the existence of energetic electrons of 30-70 $\mathrm{keV}$. The experiment shows that the suppression of $\mathrm{m} / \mathrm{n}=2 / 1$ tearing modes can be sustained by ECRH with low modulation frequency of about $10 \mathrm{~Hz}$. Extended confinement improvement is obtained after the mode suppression.
\end{abstract}

\section{Introduction}

Recent HL-2A tokamak experiment programme is to address key physics issues relevant to ITER. In accordance with the goal, HL-2A is equipped with extensive and advanced diagnostics, a digital control system, various fuelling techniques (pellet injection, gas puffing, SMBI), NBI (1.5 MW/45 keV), ECRH (2 MW/68 GHz) and LHCD (1 MW/2.45 GHz) heating systems. The achieved operation parameters of HL-2A $(\mathrm{R}=1.65 \mathrm{~m}, \mathrm{a}=0.4 \mathrm{~m})[1]$ are as follows: toroidal magnetic field is $2.7 \mathrm{~T}$, plasma current $450 \mathrm{kA}$, electron temperature 5 $\mathrm{keV}$, ion temperature $1.5 \mathrm{keV}$, and electron density $(1-9) \times 10^{19} \mathrm{~m}^{-3}$. The experimental campaigns in recent years have been focused on studying and understanding the physics of zonal flow and turbulence, transport, MHD instabilities and energetic electron dynamics. This overview describes the main physics results in those fields obtained since the last Fusion 
Energy Conference. The achievements benefit from the following hardware development and improvement. Diagnostics with high spatial and temporal resolution include: Thomson scattering for Te, five soft x-ray arrays for MHD study, microwave reflectometer for electron density profile and Doppler reflectometry for plasma rotation and core turbulence measurements, specially designed Langmuir probes for 3D zonal flow and edge turbulence measurement, 10-channel CdTe hard x-ray detector for energy distribution of energetic electrons. Besides, the supersonic molecular beam injection (SMBI) system has been improved, the modulation frequency of SMBI reaches $50 \mathrm{~Hz}$, the system is not only an advanced technology for fuelling, but also a powerful tool (combined with microwave reflectometer) for particle transport study. The NBI system with four ion sources (power up to 1.5 MW) has been commissioned and operated on HL-2A.

\section{Transport}

\subsection{Spontaneous internal particle transport barrier}

Particle transport barriers with energy confinement improvement have been observed in tokamaks, at the edge of the H-mode plasmas [2] as well as in the core (ITB) in advanced discharges [3-5]. These regimes have often been achieved with high power RF wave or neutral beam heating. On HL-2A tokamak, natural or spontaneous particle transport barriers were observed in purely ohmically heated plasmas without any external momentum input or extra particle sources.

In the HL-2A experiments, internal particle transport barriers (pITBs) were found to be formed in ohmic plasmas when the electron density increases to a critical value [6]. Fig.1 shows the temporal evolution of the line-averaged density profile for a representative discharge with transition to a pITB. Before $\mathrm{t}=420 \mathrm{~ms}$, no clear barrier had been observed. Starting from this moment, a pITB developed gradually around $\mathrm{r}=29 \mathrm{~cm}$. The critical line-averaged electron density corresponding to this transition is $\mathrm{n}_{\mathrm{c}}=2.2 \times$ $10^{19} \mathrm{~m}^{-3}$. The pITB domain is from $\mathrm{r}=28 \mathrm{~cm}$ to $\mathrm{r}=30 \mathrm{~cm}$. The width of the barrier is $1-2 \mathrm{~cm}$. There is a drastic change in the density gradient through the barrier: $\mathrm{L}_{\mathrm{n}} \sim 10 \mathrm{~cm}$ inside the barrier, $\mathrm{L}_{\mathrm{n}} \sim 50 \mathrm{~cm}$ outside the barrier and $\mathrm{L}_{\mathrm{n}} \sim 25 \mathrm{~cm}$ in the core and edge regions.

To study the transport features of the pITB, the density modulation generated by SMBI has been used [7]. Fig. 2(a) and (b) display the phase and the amplitude of the 1st Fourier harmonic of the modulated density. In the experiment, the SMBI modulation frequency is

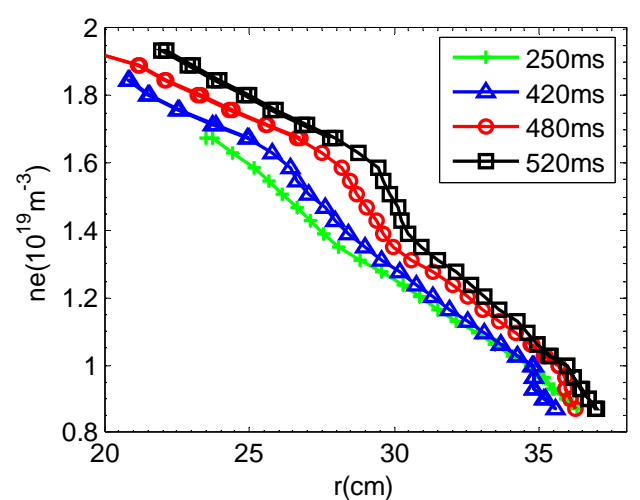

Fig.1. Shot \#7557. Temporal evolution of the density profile at selected moments measured by a broadband O-mode reflectometer. Before the appearance of the barrier (250 ms); at the beginning of the appearance of the barrier (420 ms); strong barrier (480 ms); just after a particle pulse injection by SMBI (520 ms). 
$9.6 \mathrm{~Hz}$, the duration of SMBI pulse is about $6 \mathrm{~ms}$, the gas pressure of SMBI is $1.3 \mathrm{MPa}$. The simulation with an analytical model [8] for particle transport was carried out to characterize this barrier quantitatively. In Fig. 2 (a) and (b), the solid lines represent the simulation results,
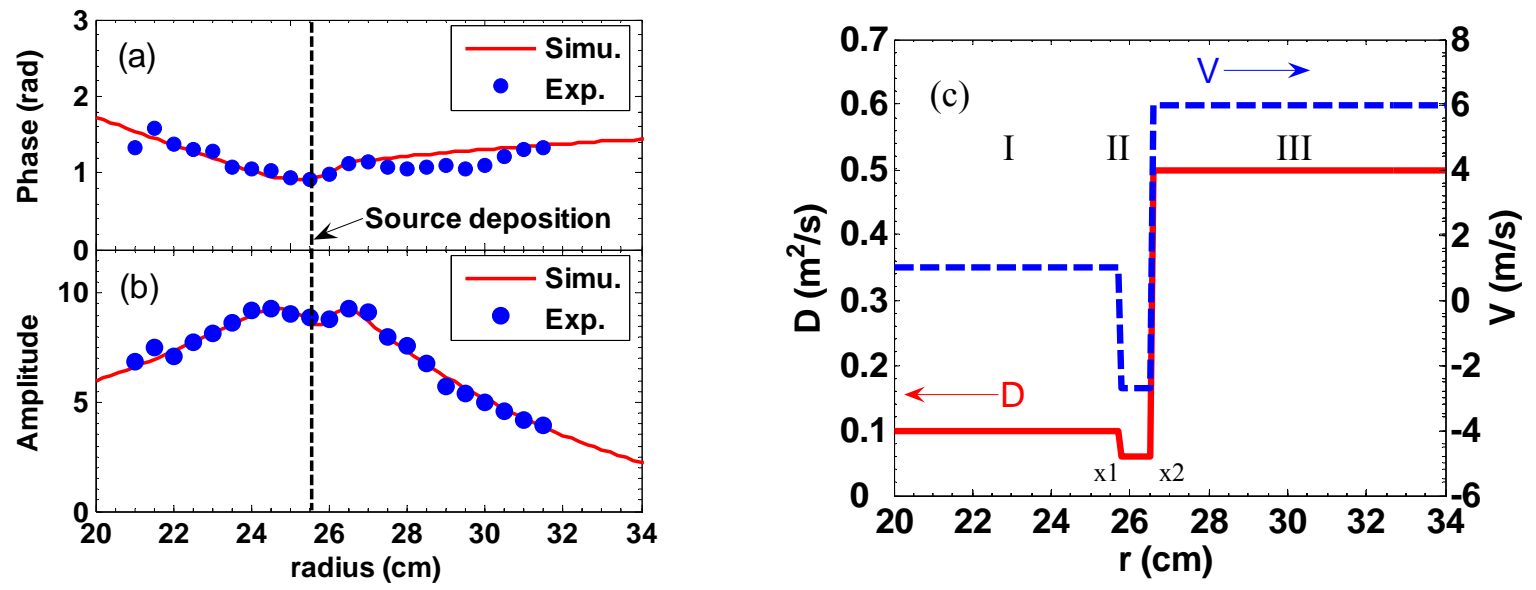

Fig. 2. Shot \#7593. Phase (a), amplitude (b) of the 1st harmonic of the Fourier transform of the modulated density. (c). Diffusivity $D$, convective velocity $V$ for the simulation

solid circles are experimental results. A satisfactory agreement has been found between the experiments and the analytical calculation. It can be seen from Fig. 2(c) that $D_{1}=0.1 \mathrm{~m}^{2} / \mathrm{s}$ and $\mathrm{V}_{1}=1.0 \mathrm{~m} / \mathrm{s}$ in the domain I $\left(\mathrm{r}<\mathrm{x}_{1}\right), \mathrm{D}_{2}=0.045 \mathrm{~m}^{2} / \mathrm{s}$ and $\mathrm{V}_{2}=-2.7 \mathrm{~m} / \mathrm{s}$ in the domain II $\left(\mathrm{x}_{1}<\right.$ $\left.\mathrm{r}<\mathrm{x}_{2}\right), \mathrm{D}_{3}=0.5 \mathrm{~m}^{2} / \mathrm{s}$ and $\mathrm{V}_{3}=6.0 \mathrm{~m} / \mathrm{s}$ in the domain III $\left(\mathrm{r}>\mathrm{x}_{2}\right)$. In comparison, a SMBI modulation experiment has also been performed for a discharge with a density $\left(\mathrm{n}_{\mathrm{e}}=1.9 \times\right.$ $10^{19} \mathrm{~m}^{-3}$ ) lower than the critical density $\mathrm{n}_{\mathrm{c}}$. In this case, no barrier has been observed, the

diffusivity obtained by the method is $D=0.25 \mathrm{~m}^{2} / \mathrm{s}$ for $\mathrm{r}=(28-31) \mathrm{cm}$, and a negative convective velocity has been found $V=-2.2 \mathrm{~m} / \mathrm{s}$ for $\mathrm{r}=(28-31) \mathrm{cm}, \mathrm{V}=-4.2 \mathrm{~m} / \mathrm{s}$ for $\mathrm{r}=$ (31-33) $\mathrm{cm}$. From the density modulation experiments, it is found that the convective velocity is negative when the density is lower than the threshold and positive when the density exceeds the threshold, except for inside the barrier where the particle convective velocity remains negative. The diffusivity $\mathrm{D}$ is rather well-like than step-like.

Fig. 3 shows the radial profiles of the turbulent poloidal rotation velocity of the plasma measured with the Doppler reflectometry [9] at different densities. When the electron density is lower than the critical density $n_{c}=2.2 \times 10^{19} \mathrm{~m}^{-2}$, the measured velocity increases monotonously in the

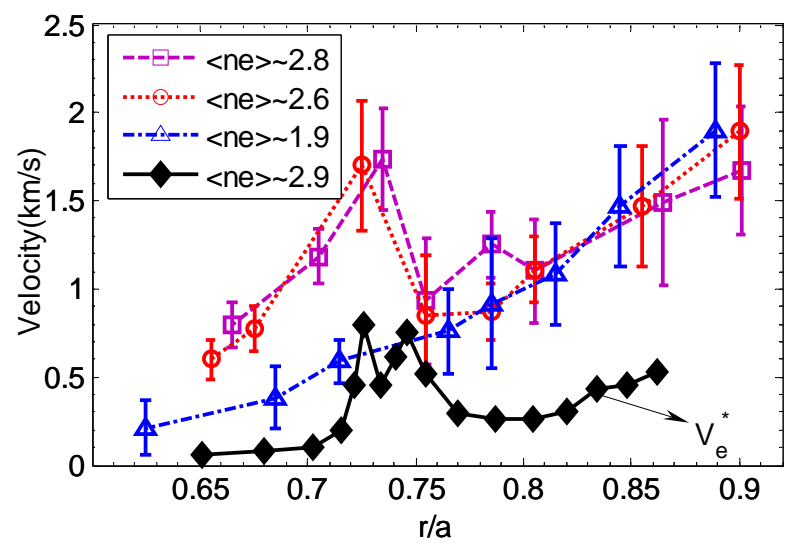

Fig.3. Radial profile of the turbulent poloidal rotation velocity with pITB $(\bigcirc, \square)$ and without pITB $(\Delta)$ measured by Doppler reflectometry. Radial profile of the electron diamagnetic drift velocity $V_{e}^{*}$. 
region of $0.6<\mathrm{r} / \mathrm{a}<0.9$. When the density exceeds the density threshold, a drastic change in the measured rotation velocity is observed in the barrier region $r / a=0.6-0.7$. The electron diamagnetic drift velocity $V_{e}^{*}$ is also given in Fig. 3, which is computed from the measured density and electron temperature profiles. The same feature is observed in the barrier region for $V_{e}^{*}$, assuming $V_{\perp}=V_{E \times B}+V_{e}^{*}, V_{E \times B} \approx 1 \mathrm{~m} / \mathrm{s}$ for $0.6<\mathrm{r} / \mathrm{a}<0.9$. This may suggest that the drastic change observed in the turbulent rotation velocity at the barrier results from the steepness of the density gradient in the barrier. Because the Doppler reflectometry detection system is homodyne, the turbulent direction is not available with the diagnostics.

The change of the sign for the convective velocity may be explained with the turbulence TEM/ITG regimes $[10,11]$. The density threshold may be correlated to the TEM/ITG transition via the collisionality. However the mechanism leading to the pITB formation remains unclear. On the other hand, the formation of the barrier coincides with the TEM/ITG transition. Thus it is not excluded that the transport barrier is created initially by the discontinuity or jump in the convective velocity during the TEM/ITG transition, or more precisely, by two convective velocities in opposite direction (inward/outward) at the barrier. This speculation needs, of course, further experiments to be confirmed.

\subsection{Non-local transport with SMBI}

Since the first observation of the non-local effect on TEXT-U in 1995 [12], different edge cooling techniques [13-16] have been widely used in studying the effect: impurity injection by laser ablation, ice pellet injection, carbon-based molecules injection, etc. On HL-2A, non-local effect has been observed after supersonic molecular beam injection.

The results on the non-local effect induced by SMBI on HL-2A show some new features: the duration of the core $\mathrm{Te}$ rise could be prolonged by changing the condition of the injected SMBs (see Fig. 4); both the thermal radiation and the $\mathrm{H} \alpha$ emission decrease when the non-local effect appears [16]. Fig. 5 shows the Fourier transformation of the electron temperature measured by ECE when the Te modulation was induced by the modulated SMBI at $\mathrm{f}=10 \mathrm{~Hz}$. For all harmonics a strong

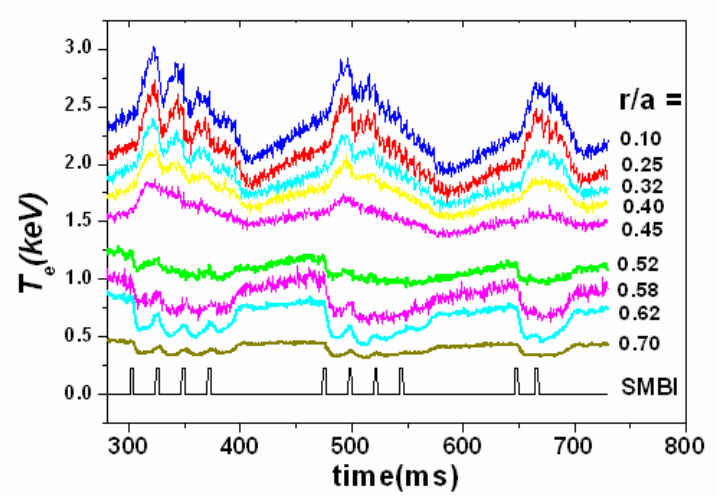

Fig. 4. Shot 8364. The temporal evolution of Te measured by the ECE at different radii. The sustainment of the core Te rise induced by $S M B I$ is prolonged by changing the time separation between two SMB pulses. It is 20 $\mathrm{ms}, 20 \mathrm{~ms}$ and $15 \mathrm{~ms}$ for three successive events respectively. The pulse duration is $4 \mathrm{~ms}$. decrease in amplitude and a clear phase jump occur at $\mathrm{r} \sim-18 \mathrm{~cm}$, which is the reverse position of the electron temperature change. Two peaks in amplitude profile and two corresponding troughs in phase profile can be found for all harmonics. Those may indicate that two perturbation sources exist in the regions outside and inside the inversion radius, respectively. The position of 
the outer initial heat pulse is found to be at $\mathrm{r} \sim-25 \mathrm{~cm}$, which depends on the deposition location of SMBI, and the behaviour of the perturbation shows usual propagation features of SMBI cold pulse. The value of $\chi_{\mathrm{e}} \mathrm{HP}$ deduced from Fourier analysis, showing agreement with the result from sawteeth pulse propagation, is in the range $(2-3) \mathrm{m}^{2} / \mathrm{s}$. In the inner region, the initial perturbation is found to be in the core, yielding a faster outward propagation, and $\chi_{\mathrm{e}} \mathrm{HP}$ is in the range $(6-8) \mathrm{m}^{2} / \mathrm{s}$. Profiles of amplitude and phase at the first three harmonics are independent of the SMBI modulation frequency. The steeper profiles around the interface, which means a reduction of heat transport, indicate that an internal transport barrier is formed in this region. With the good flexibility and easy controllability of the SMBI conditions (gas pressure, modulation frequency, duty cycle, etc.), modulated SMBI has been proved to be an effective tool to study the non local effect. Further investigation on the mechanism of the effect is to be undertaken on HL-2A.
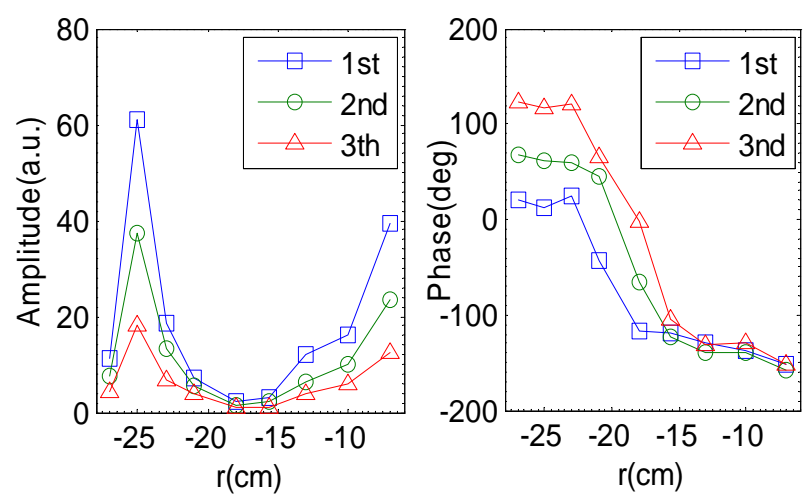

Fig. 5. The amplitudes and phases of the first three harmonics after FFT of the Te measured by ECE (shot 8337, $B_{t}=1.4 \mathrm{~T}, n_{e}=0.7-1.0 \times 10^{19} \mathrm{~m}^{-3}, I_{p}$ $=180 \mathrm{k}$.).

\section{Zonal flow andTurbulence}

\subsection{Low frequency zonal flows}

Zonal flows became a concerned issue over the last decade. Many theory predicted characteristics have been confirmed in experiments, especially for the geodesic acoustic mode (GAM) [17], which has a higher frequency and could be identified more easily. The symmetry feature of the low-frequency Zonal Flow (LFZF) [18] in the core plasma has been proved on the DIII-D by the beam emission spectroscopy [19] and on the CHS by the heavy ion beam probes [20] respectively. However, few convincing experimental evidences have been demonstrated for the existence of the LFZF in the edge plasma. In the experiments on HL-2A, the coexistence of the LFZF and GAM was observed, and the complete 3-D spectral structure of the LFZF in the edge plasma was investigated [21].

The experiments were carried out in Ohmic deuterium plasmas with a limiter configuration under the following conditions: $\mathrm{B}_{\mathrm{t}}=1.4 \mathrm{~T}, \mathrm{Ip}=160-180 \mathrm{kA}, \mathrm{q}_{\mathrm{a}}=3.5-4, \overline{\mathrm{n}}_{\mathrm{e}}=$ $(1-3) \times 10^{19} \mathrm{~m}^{-3}$. Four Langmuir probe arrays were used to measure the floating potential fluctuations $\left(\phi_{\mathrm{f}}\right)$, two of them separated by $5 \mathrm{~cm}$ poloidally and two by $130 \mathrm{~cm}$ toroidally for determining the poloidal (m) and toroidal (n) mode numbers, respectively. These measurements were located at the radius $\mathrm{r} / \mathrm{a}=0.90$.

The spectra of coherent coefficients and mode numbers between two potential fluctuations measured by the poloidally and toroidally large separated probe arrays were 
given in Figs. 6(a) and 6(b), showing that over a large frequency range from $0.5 \mathrm{kHz}$ to $\sim 15$ $\mathrm{kHz}$, the correlation coefficients are high and the mode numbers are round zero. The coherent mode at $8.5 \mathrm{kHz}$ has been identified as the GAM [22]. The low frequency (LF) feature with the potential structure of $m=n=0$ is consistent with the theoretical prediction and simulation result for the LFZF [23].
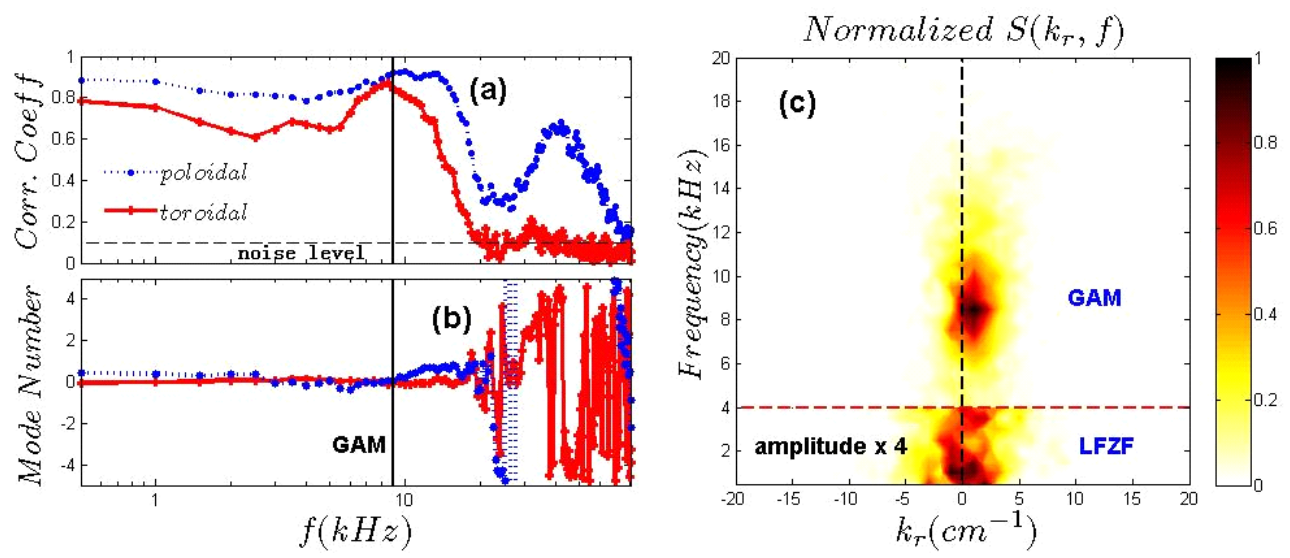

Fig. 6. (a) Coherent coefficients and (b) mode numbers between two potential fluctuations separated poloidally and toroidally. (c) Normalized wavenumber-frequency spectra below $20 \mathrm{kHz}$ (the area under the horizontal dashed line is multiplied by 4 for clarity).

The radial wave-number via frequency spectrum $S\left(k_{r}, f\right)$ for the LF feature has two peaks at approximately symmetrically positive and negative values $\bar{k}_{r} \approx \pm 1.0 \mathrm{~cm}^{-1}$ with a small imbalance in the positive and negative components. This observation implies that the energy of the LF feature propagates in the inward and outward radial directions with a net outward, as shown in Fig.6(c). The spectrally averaged wavenumber and its width are $\bar{k}_{r}=0.55 \mathrm{~cm}^{-1}$ and $\Delta k_{r}=3.2 \mathrm{~cm}^{-1}$, which corresponds to $k_{r} \rho_{i}=0.033$ and $\Delta k_{r} \rho_{i}=0.19$ respectively. This is in the range for the LFZF expected by theory and simulations [24]. The envelope analysis and bispectral approach are used to investigate the nonlinear interaction among the LFZF, GAM and ambient turbulence (AT). The results manifest the modulation of the AT envelope correlating with $\phi_{f}$ in the whole low frequency range below the GAM and the phase shifts are all around $\pi$. These clearly indicate that the AT envelope is modulated by both the LFZF and GAM, and their interaction has anti-phase relation, which suggests that both the LFZF and GAM are generated in the energy-conserving nonlinear interaction with the AT.

\subsection{The density fluctuation of GAM frequency}

The density fluctuations with typical GAM frequency of $\mathrm{f}_{\mathrm{GAM}} \sim 9.8 \mathrm{kHz}$ are observed at the radius $\mathrm{r} / \mathrm{a}=0.95$ on $\mathrm{HL}-2 \mathrm{~A}$, whose main characteristics have been identified with novel 
combination of the two probe arrays [25]. The clear peaks at the GAM frequency appear simultaneously, not only in potential fluctuations, but also in density fluctuations. The relative amplitude of the GAM density fluctuation near outer midplane is estimated as $0.02 \%$, which is derived from the higher frequency ambient turbulence (HFAT) envelope amplitude $\tilde{n}_{e n, G A M} / \widetilde{n}_{e}=0.4 \%$ at $(100-450) \mathrm{kHz}$ multiplied by the total density fluctuation $\tilde{n}_{e} / \bar{n}_{e}=5 \%$, while it is predicted as $\widetilde{n}_{\mathrm{GAM}}^{\mathrm{th}} / n_{e}=-\sqrt{2} k_{r} \rho_{i}\left(e \widetilde{\phi} / T_{e}\right) \sin \theta=0.03 \%$ by the GAM theory using the following parameters: $k_{r} \rho_{i}=0.07, e \widetilde{\phi} / T_{e}=6.2 \%$, and $\theta=2.7^{0}$. The experiments are in agreement with the theoretical prediction. The contour of poloidal and toroidal mode numbers, derived from wave number-frequency spectrum $S(k, f)$, is shown in Fig. 7. It is clear that the peak of $\mathrm{S}\left(\mathrm{m}, \mathrm{n}, \mathrm{f}_{\mathrm{GAM}}\right)$ spectrum mainly localizes at $\mathrm{m}=0.5 \sim 1.5$ and $\mathrm{n}=-0.01 \sim 0.02$. The corresponding mode numbers are estimated as $\mathrm{m}$ $=1.2 \pm 0.4, \mathrm{n}=0.036 \pm 0.039$, respectively. In another discharge without simultaneous potential measurement, the mode numbers of the GAM density fluctuations are estimated to be $m=$ $-0.73 \pm 0.65$ and $n=0.035 \pm 0.04$. The results are close

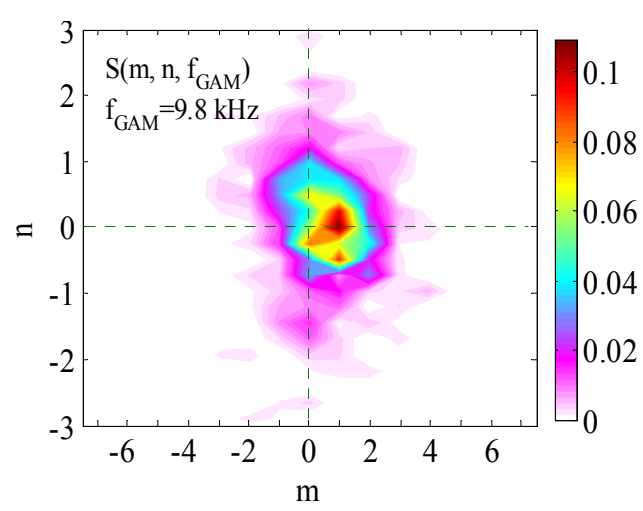

Fig. 7. The contour of $S\left(m, n, f_{G A M}\right)$ with $I_{s}$, estimated by two-point technique. to the mode numbers $(\mathrm{m}=0, \mathrm{n}=0)$ of theoretical prediction for GAM density fluctuations. The mode numbers for potential fluctuation are estimated as $\mathrm{m}=0.23 \pm 0.36$ and $\mathrm{n}=0.04 \pm 0.04$ with the same technique.

The nonlinear coupling between the GAM and ambient turbulence is explored with bispectral analysis, as indicated in Fig. 8. The auto-bicoherence of saturation ion current shows a noticeable peak under the conditions of $\mathrm{f}_{1}+\mathrm{f}_{2}=9.8 \mathrm{kHz}$ and $30<\mathrm{f}_{1}<250$ $\mathrm{kHz}$, which implies strongly nonlinear interactions between the GAM and ambient turbulence. The summed bicoherence under the fixed frequency $\mathrm{f}_{3}=\mathrm{f}_{\mathrm{GAM}}$ has also a peak with the $\mathrm{f}_{\mathrm{GAM}}=9.8 \mathrm{kHz}$, which suggests that three-wave interaction is a plausible mechanism to generate the GAM and the driving energy comes from small scale drift
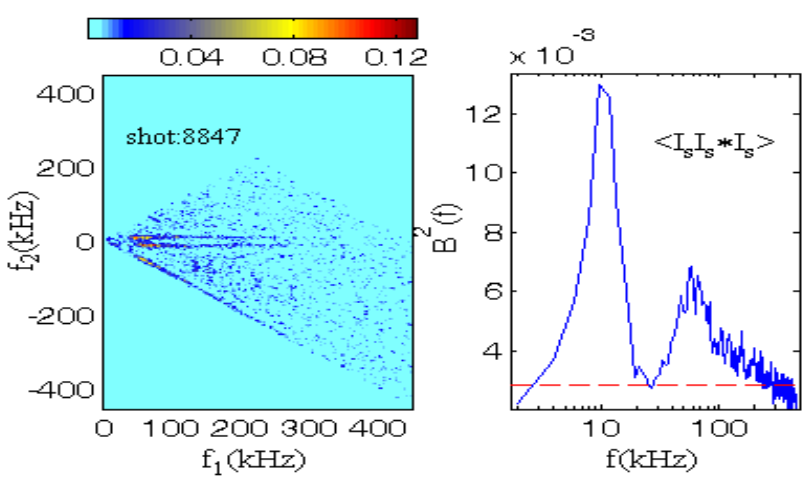

Fig.8. (a) The contour of the squared auto bicoherence $b^{2}\left(f_{1}, f_{2}\right)$, (b) the summed bicoherence $b^{2}$. The noise level is $2.5 \times 10^{-3}$, indicated by red dotted line.

fluctuations. It is noticed that there is another broad-peak $(30-80 \mathrm{kHz})$ in the summed bicoherence $b^{2}(f)$, which is possibly correlated with quasi-mode (QM), discussed in section 3.3.

In addition, the phase shifts between density fluctuations and the envelope of high 
frequency components $(200-450 \mathrm{kHz})$ of the AT at the GAM frequency are close to $0.9 \pi$, which can be considered to be an indicator for envelope modulation. This observation suggests that GAM can regulate the ambient turbulence through envelope modulation.

\subsection{Quasi-modes-like low frequency fluctuations}

Recently, simulation and theory indicate that intermediate scale quasi-modes (QM) also play an important role in determining saturation level of the turbulence and corresponding transport, especially, in cases of the absence of zonal flows such as the turbulence driven by electron temperature gradient (ETG) [26-28].

Fig. 9 shows the typical auto-power spectra of the floating potential fluctuation measured on HL-2A [29]. The first peak centered at frequency of $\sim 1 \mathrm{kHz}$, corresponding to the MHD modes and LFZF. The toroidal symmetry of the second peak at GAM frequency of $\sim 9 \mathrm{kHz}$ and its interaction with the AT are discussed in detail in previous papers $[30,31]$. The third peak centered at the frequency of $\sim 35 \mathrm{kHz}$ is the low frequency fluctuations or quasi-mode (LFF or $\mathrm{QM})$ with the width $\Delta \mathrm{f} / \mathrm{f}=0.7$. The turbulence of broad frequency in $(100-500) \mathrm{kHz}$ without apparent spectral peak is considered as the higher frequency ambient turbulence (HFAT) in

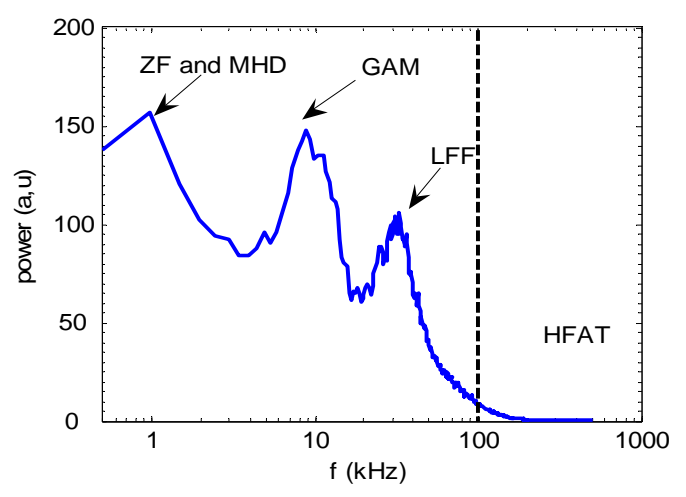

Fig. 9. Typical auto-power spectra of floating potential fluctuation. comparison. The spectral width of the LFF is estimated as (20-40) $\mathrm{kHz}$, corresponding to the lifetime of (25-50) $\mu \mathrm{s}$. The autocorrelation time of the HFAT is estimated as $\sim 5 \mu$ s from autocorrelation function analysis. It has been shown that the poloidal and toroidal correlation lengths of the LFF may reach $\sim 6.5 \mathrm{~cm}$ and $\sim 80 \mathrm{~cm}$ and are much longer along magnetic field lines than that deviated from field lines. In contrast, the estimated poloidal correlation length of the HFAT is $\sim 0.5 \mathrm{~cm}[30,31]$. These results show that the spatial and temporal scales of the LFF are about one order of magnitude higher than those of the HFAT.

The poloidal wave number spectra for three typical discharges are given in Fig. 10. Here, the poloidal wave vector spectra of the HFAT show apparent linear dispersion relation, from which deviations occur in the LFF regime. The two regimes feature of the spectrum and thus of the turbulence seems universal and reproducible even though the wave vector spectra look like different. The similar results are also obtained in radial and toroidal directions. The nonlinear three wave coupling between the LFF and the

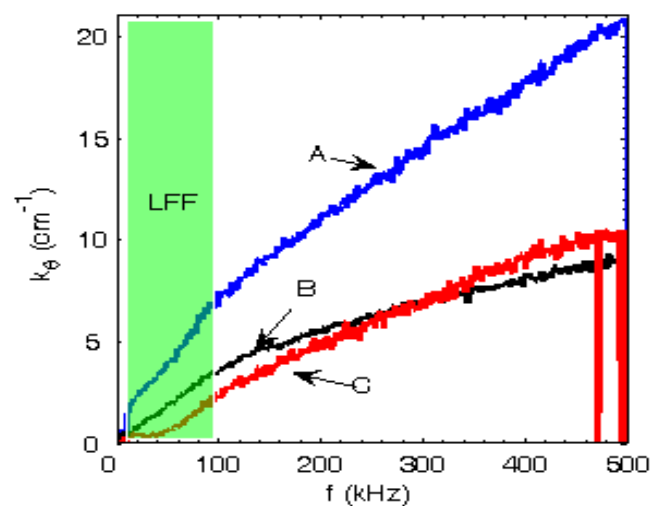

Fig. 10. Poloidal wave vector spectra for 3 typical discharge parameters. 
HFAT is identified with bi-coherence analysis to be a plausible generation mechanism for the former. The phase shift between the LFF and the envelopes of the HFAT is around $\pi$, indicating possible regulating effects of the former on the latter [31]. The LFFs have long wave lengths along magnetic field lines [30, 31]. The results are similar to the theory and simulation predictions on quasi-mode. Further analysis on this topic is in progress.

\section{MHD Activities with ECRH}

\subsection{Internal kink mode driven by energetic electrons}

Since the electron fishbone (e-fishbone) instability was observed for the first time on DIII-D [32] with both NBI and off-axis ECCD, experiments have been carried out on HL-1M [33] with ECRH, FTU [34] with LHCD and Tore Supra [35]. The instability is thought to be excited by the barely trapped electrons (BTEs) [32] or the barely circulating electrons (BCEs) at the negative magnetic shear $[36,37]$. To identify the resonance conditions, hard x-ray detectors $(\mathrm{CdTe})$ were used to measure the energetic electron spectra on HL-2A. The experimental results show that the excitation of the e-fishbone instability depends on the spectra of the energetic electrons and agree with the resonance conditions qualitatively.

Fig. 11 shows the temporal evolution of the soft x-ray emission during ECRH on the HL-2A tokomak. A strong internal kink mode has obvious features of the fishbone structure characterized by the fishbone like amplitude bursting. The instability can be excited by ECRH deposited at both the higher field and lower field sides. The mode is located around the $\mathrm{q}=1$ surface. The frequency is between 4 and $8 \mathrm{kHz}$.

According to the experiments on HL-2A [38], the mode is excited with the increase of the $35-70 \mathrm{keV}$ energetic electrons. Fig. 12 shows the hard $\mathrm{x}$-ray photon numbers versus energetic
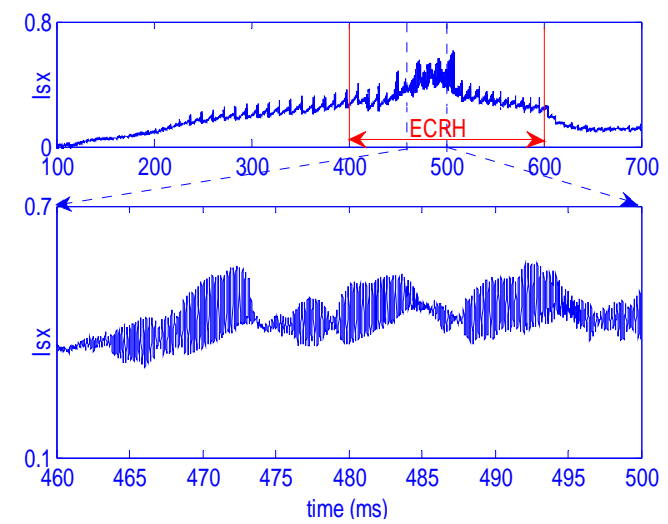

Fig. 11. The temporal evolution of the soft $X$-ray emission intensity with fishbone-like instability.

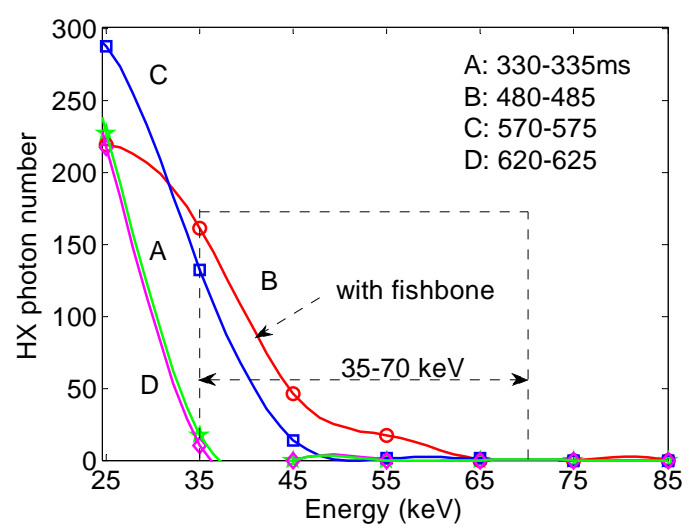

Fig. 12 .The hard $X$-ray photon numbers versus energetic electron energy at different times for Shot 7982.

electron energy at different time slices. It is shown that the mode grows with the increase of 
the $35-70 \mathrm{keV}$ energetic electrons, when the electron velocity distribution deviates from Maxwellian and the energetic electrons interact with internal kink mode. It seems that the energetic electrons with energy in the range of $35-70 \mathrm{keV}$ dominate the effect. The calculation results [38] show that the mode frequency is close to the precession frequencies of the BTEs and the BCEs. The vertical and parallel energies of the electrons resonating with the internal kink mode are in the range of (10-100) $\mathrm{keV}$ and the precession frequency is about $0-20 \mathrm{kHz}$ when the magnetic shear is very weak or negative. The fishbone modes may be excited by the BCEs with off-axis ECRH at the LFS. However, at the HFS, the modes may be driven jointly by the BTEs and BCEs.

\subsection{The stabilization of the $m=2, n=1$ tearing mode with $E C R H$}

The stabilization of tearing modes by off-axis ECRH in vicinities of the rational surfaces has been realized on tokamaks. This may lead to disruption avoidance and confinement improvement. on the other hand, it has been observed that the core electron temperature keeps constant for some time just after off-axis ECRH switch-off, which suggests that the transport coefficients may be reduced in the core [39]. These suggest the possibility of applying off-axis ECRH in an integrated way to combine the effects on both the stabilization of tearing mode and the reduction of the transport.

The investigation on the suppression of $m=2 / n=1$ tearing mode by ECRH has been performed on HL-2A. The toroidal magnetic field is adjusted so that the $68 \mathrm{GHz} \mathrm{EC}$ resonance occurs at different positions with respect to the $\mathrm{q}=2$ surface at the low field side. It is found that when the location of the EC resonance is close to the $\mathrm{q}=2$ surface, the tearing mode is suppressed, as shown in Fig.13. With the suppression, clear increases of the plasma density and stored energy are observed, and a transient ITB is obtained after ECRH switch-off in the experiments with the ECRH power in the range of $0.6<\mathrm{P}_{\mathrm{ECRH}} / \mathrm{P}_{\mathrm{ohm}}<1$. The interesting feature of the ITB appearance after ECRH switch-off and the fact that such kind of effect can be additive [40, 41], has motivated us to apply successive ECRH pulses for sustaining MHD-free phase and obtaining continuous confinement improvement.

As is shown in Fig.14, a better suppression can be achieved with lower frequency modulation and
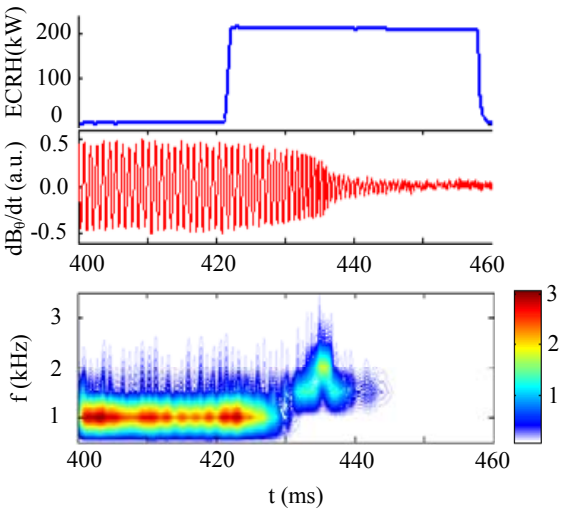

Fig.13 Time traces of magnetic fluctuation and its spectrogram for a discharge in which ECRH is applied far off-axis near the $q=2$ surface. appropriate deposition position of the ECRH power,. The tearing mode is effectively suppressed by the successive ECRH in the vicinity of the $\mathrm{q}=2$ surface (the blue lines) in contrast to the poor suppression or even driving of the mode with inappropriate power deposition position about $3 \mathrm{~cm}$ inside the $\mathrm{q}=2$ surface (the red lines). The suppression event is characterized by the continuous improvement of 
confinement, i.e. the plasma density, temperature, stored energy and energy confinement time increase steadily throughout the successive ECRH pulse. The continuous rise in central plasma density and temperature as well as the confinement improvement, may be attributed to the additive effect of the internal transport barrier formed after each ECRH pulse switch-off. Therefore, successive ECRH pulses may be applied to explore a more favorable regime taking advantage of the enhanced confinement due to a series of ITB formation. This may provide an effective means of controlling $\mathrm{m} / \mathrm{n}=2 / 1$ tearing modes and extending the beneficial effects of MHD suppression on particle and energy confinement.

\section{Future plan}

Significant advances in fusion science studies have been made on the HL-2A device, aided by the development and

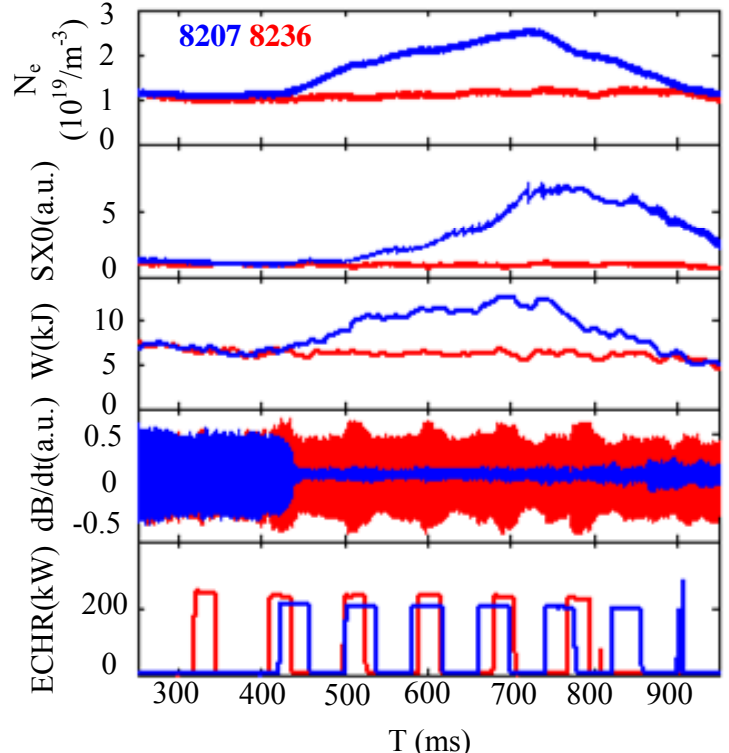

Fig.14 Time traces for discharge 8207 in which several successive ECRH pulses produce an additive increase in density, SXR emissivity and stored energy. Also shown is a case with 2/1 tearing mode when ECRH power is inside the $q=2$ surface (8236). installation of the hardware including diagnostics such as high resolution ECE, multichannel HCN laser interferometer, and heating systems of $2 \mathrm{MW}$ ECRH/ECCD, 1.5 MW NBI, etc.. In addition, a prototype of CXRS has been developed and tested, a multichannel NPA system will be applied in November this year. Besides, further hardware developments are underway. Auxiliary heating system will be extended, another beam line of NBI and ECRH system (140 $\mathrm{GHz}$ ) will provide more capability for physics study. The following diagnostics will also be designed and commissioned in the near future: an MSE system for the plasma current profile measurement, an ECEI for 2D visualization of MHD activity, $\mathrm{CO}_{2}$ laser scattering for bulk plasma turbulence. These developments in conjunction with development of theory and simulation software will enhance the capability of HL-2A for the experimental investigation of the key science and technology subjects relevant to ITER and fusion DEMO. The short term physics program will focus on MHD activities; RF wave heating and current drive, including mechanism, efficiency and control; H-mode physics, including formation mechanism or trigger, pedestal characteristics and edge localized modes; particle, momentum and energy transports and confinements: including micro-instabilities, turbulence and zonal flows, ITB formation mechanism emphasizing the role played by the rational magnetic surfaces, the magnetic and flow shears; SOL and divertor physics, including blob, turbulence and transport in SOL, particle and heat loading on the plasma facing components, and plasma-materials interactions.

\section{References}


[1] Y. Liu et al., 2005 Nucl. Fusion 45239.

[2] F. Wagner, G. Faussmann, D. Grave et al., Phys. Rev. Lett. 53, 1453 (1984).

[3] M.J. Greenwald, D.A. Gwinn, S. Milora et al., Phys. Rev.Lett. 53, 352 (1984).

[4] C.L. Fiore, J.E. Rice, P.T. Bonoli et al., Phys. Plasmas 8, 2023 (2001).

[5] E.J. Doyle, G.M. Staebler, L.Zeng et al., Plasma Phys. Controlled Fusion 42, A237 (2000).

[6] W.W. Xiao, X.L. Zou, X.T. Ding, et al., 2008, IAEA-EX/P5-24.

[7] L.H. Yao et al., Nucl. Fusion 38, 631 (1998).

[8] S.P. Eury, E. Harauchamps, X.L. Zou et al. Phys. Plasmas 12, 102511 (2005).

[9] X.L. Zou, T.F. Seak, M. Paume et al., Proc. 26th EPS Conf. on Controlled Fusion and Plasma Physics (Maastricht) Vol. 23J, p.1041 (1999).

[10] X. Garbet, L. Garzoti, P. Mantica et al., Phys. Rev. Lett. 91, 035001 (2003).

[11] C. Bourdelle, X. Garbet, F. Imbaux et al., Phys. Plasmas 14, 112501 (2007).

[12]J. D. Callen, Phys.Fluids B, 4 (1992) 2142.

[13] K. W. Gentle, et al., Phys. Rev. Lett. 74 (1995) 3620.

[14] G. M. D. Hogeweij, et al., Plasma Phys .Contr. Fusion 42 (2000) 1137.

[15] X.L. Zou, et al., Plasma Phys. Control. Fusion 42 (2000) 1067.

[16] H.J. Sun, et al., IAEA-EX/P5-23.

[17] N.Winsor et al., Phys. Fluids 11, 2448 (1968).

[18] Z.Lin et al., Science, 281, 1835(1998).

[19] G. McKee et al., Phys. Plasmas, 10, (2003), 1712.

[20] A. Melnikov et al., Plasma Phys. Control. Fusion, 48, (2006), S87.

[21] A.D.Liu et al., 2008 IAEA-EX/P5-23.

[22] T. Lan et al., Phys. Plasmas 15, 056105 (2008).

[23] P. H. Diamond et al., Plasma Phys. Control. Fusion 47, R35 (2005).

[24] T. S. Hahm et al., Plasma Phys. Control. Fusion 42, A205 (2000).

[25] L.W. Yan et al., 2008 IAEA-EX/P5-33.

[26] L.Chen, F Zonca and Z.Lin, Plasma Phys. Control. Fusion, 47, B71 (2005).

[27] Z.Lin, L.Chen and F.Zonca, Phys.Plasmas, 12, 056125 (2005).

[28] W.X.Wang et al., Phys.Plasmas, 14, 072306 (2007).

[29] K.J.Zhao et al., 2008, IAEA-EX/P5-34.

[30] K.J.Zhao et al., Phys. Rev. Lett. 96, 255004 (2006).

[31] K.J.Zhao et al., Phys.Plasmas, 14,122301 (2007).

[32] K L Wong et al 2000 Phys. Rev. Lett. 85996.

[33] X T Ding et al 2002 Nucl.Fusion 42491.

[34] P Smeulders et al 2002 Fast MHD Analysis on FTU, Proc. 29th EPS Conf. on Pl. Phys. Contr. Fusion (Montreux, 2002).

[35] P Maget et al 2006 Nucl.Fusion 46797.

[36] Z T Wang et al 2007 Nucl. Fusion 471307.

[37] F Zonca et al 2007 Nucl.Fusion 471588.

[38] W Chen et al., 2008, IAEA-EX/P8-11.

[39] K.A.Razumova et al., Nucl.Fusion 44 (2004) 1067.

[40] Yunbo Dong, Yi Liu et al., 2008, IAEA-EX/P3-8.

[41] J.D. Callen et al., Plasma Phys. Control Fusion 39 (1997) B173. 